\title{
Comunidad de Escarabajos Coprófagos (Coleoptera: Scarabaeidae: Scarabaeinae) en Hábitats bajo Distinta Intensidad de Uso en Yuscarán, Honduras
}

\author{
José D. Rivera ${ }^{1}$ y Karla J. Cantarero ${ }^{2}$
}

Resumen: Los recursos naturales en el Municipio de Yuscarán se encuentran bajo un continuo proceso de degradación producto de mal manejo del suelo, así como prácticas agrícolas y ganaderas intensivas. Los escarabajos coprófagos (Coleoptera: Scarabaeidae: Scarabaeinae) son insectos muy susceptibles a la perturbación del hábitat; por lo tanto, el objetivo de la investigación fue describir como la estructura de sus comunidades es modificada desde hábitats menos a más intervenidos, a través de diferentes usos del suelo, tomando en cuenta la intensidad de uso y su estructura vegetal. La investigación se realizó de junio a diciembre del 2012 en un Bosque Tropical Siempre Verde y un Arbustal Deciduo de Tierras Bajas, cada uno con dos zonas divididas en tres sitios de muestreo según el uso del suelo. Los escarabajos se recolectaron utilizando trampas de caída cebadas con estiércol de vaca. Se caracterizó la vegetación y se registraron parámetros abióticos por hábitat. Se capturaron 2,749 individuos pertenecientes a 9 tribus, 11 géneros y 21 especies. La abundancia promedio de escarabajos disminuyó más del 50\% al intensificarse la intervención humana. En el Bosque Siempre Verde, las comunidades de escarabajos estuvieron conformadas principalmente por especialistas y generalistas forestales; en el Arbustal Deciduo, las comunidades estaban compuestas por especies generalistas y heliofílicas. Este estudio muestra como la intervención humana conlleva a la pérdida de riqueza, diversidad y abundancia de las comunidades de escarabajos del Bosque Siempre Verde. No obstante, la situación es inversa y más precaria en el Ecosistema Deciduo, donde los resultados indican que se puede estar llegando a una homogenización de fauna con hábitos generalistas producto del alto grado de intervención del hábitat.

Palabras clave: Diversidad alfa, diversidad beta, estructura de comunidades, gremios, intervención humana, uso de suelo, Scarabaeinae.

\section{Dung Beetle (Coleoptera: Scarabaeidae:Scarabaeinae) Community in Habitats under Different Usage Intensity in Yuscarán, Honduras}

\begin{abstract}
The Municipality of Yuscarán is under continuous habitat degradation processes due to invasive agriculture and cattle practices. Dung beetles (Coleoptera: Scarabaeidae: Scarabaeinae) are sensitive to habitat disturbance. The objective of this study was to describe how the dung beetle community structure is modified from less to more perturbed habitats throughout three different landscapes, according to the intensity of land use and vegetation structure. The investigation took place from June to December 2012 in a Tropical Evergreen Pine Forest and in a Deciduous Woodland. Each forest type consisted of two different areas divided by 3 collection sites according to land use. Beetles were collected in pitfall traps baited with cow dung. Vegetation structure and abiotic parameters were determined per habitat. A total of 2,749 beetles were captured, belonging to 9 tribes, 11 genera, and 21 species. Mean beetle abundance was reduced by $50 \%$ due to increased human intervention. In the Evergreen Forest, the dung beetle community structure was mainly formed by forest specialists and generalists, whereas in the Deciduous Woodland the dung beetle community was formed mainly by generalist and heliophilic species. The study shows how human intervention diminishes the richness, diversity, and abundance of the dung beetle communities in the Evergreen Forest. However, there is an inverse scenario in the Deciduous Woodland where the results indicated that there could be a faunal homogenization of generalist beetles, biased towards degraded and highly perturbed habitats.
\end{abstract}

Key Words: Alpha diversity, beta diversity, community structure, guilds, human perturbance, land use, Scarabaeinae.

\footnotetext{
${ }^{1}$ Universidad Nacional Autónoma de Honduras, Facultad de Ciencias, Escuela de Biología, Tegucigalpa, Honduras, C.A. jdr495@hotmail.com

${ }^{2}$ Universidad Nacional Autónoma de Honduras, Facultad de Ciencias, Escuela de Biología, Tegucigalpa, Honduras, C.A. karlacan25@yahoo.com
}

DOI: $10.5377 /$ ceiba.v52i2.1758 


\section{Introducción}

Honduras es un país en vías de desarrollo cuya economía depende principalmente de la agricultura y ganadería (I.N.E., 2009). La población aumenta exponencialmente cada año (CELADE/UNFPA, 2005) y causa mayor demanda por alimentos y recursos naturales, lo que conlleva a la creación de más zonas de cultivos, pastizales y usos de suelo invasivos que transforman paisajes naturales a zonas degradadas. Estos procesos son perjudiciales para la estructura, función y sostenibilidad de los ecosistemas (Halffter $e t$ al., 2001), alterando los sistemas de renovación y recambio de los recursos naturales, como la dinámica del agua, temperatura y calidad del suelo. También se compromete la biodiversidad local y regional, relaciones tróficas entre las especies y su conservación (Halfftler et al., 2001). Todos estos elementos en conjunto afectan la calidad de vida de las comunidades humanas y los pobres son los más vulnerables (Barbier y Bishop, 1995, Eitzinger et al. 2012).

El municipio de Yuscarán, ubicado en la región centro-oriental de Honduras, en el departamento El Paraíso, posee recursos bajo continua presión humana, la cual repercute negativamente en la calidad y sostenibilidad de dichos recursos (Padilla et al., 2009); gran parte de su cobertura vegetal original se fragmenta producto del avance agrícola (PAGS, 1998).

Los escarabajos coprófagos (Coleoptera: Scarabaeidae: Scarabaeinae) son un grupo conspicuo de insectos muy sensibles a los cambios en la vegetación, suelo, microclima y fragmentación, que modifican los componentes principales de sus comunidades como abundancia, diversidad y composición de gremios (Howden y Nealis, 1975; Nichols et al. 2008), considerándoles como un grupo indicador excelente de la calidad del hábitat (Spector y Forsyth, 1988). Estos realizan importantes funciones ecológicas al remover y enterrar grandes cantidades de heces para su ovoposición (Camberfort, 1991).

Entre sus funciones más importantes, los escarabajos fertilizan el suelo (Bertone et al., 2006; Yamada et al., 2007), incrementando su aeración y porosidad (Brown et al., 2010). Son dispersores secundarios de semillas (Estrada y Coates-Estrada, 1991; Vulinec, 2002; Andresen, 2003), ayudan a promover el crecimiento del pasto (Bang et al., 2005; Arnaudin, 2012) y suprimen organismos patógenos del suelo en los pastizales (Doube et al., 1991).

En el Municipio de Yuscarán existe un desconocimiento general sobre la diversidad y estado de conservación de Scarabaeinae, este es el primer estudio cuyo objetivo es describir como la estructura de sus comunidades es alterada en tres paisajes bajo distinta intensidad de uso según su riqueza, diversidad y composición de gremios, contrastando las comunidades de dos ecosistemas, un Bosque Siempre Verde y un Arbustal Deciduo.

\section{Materiales y Métodos}

Área de estudio: Yuscarán, ubicada en el departamento de El Paraíso, tiene una topografía accidentada e irregular, los rangos altitudinales de la zona varían de 400 a $1900 \mathrm{msnm}$. La precipitación oscila de $1800 \mathrm{~mm}$ a $1300 \mathrm{~mm}$ por año (Argeñal, 2011). El estudio se realizó en dos ecosistemas diferentes de Yuscarán, con dos zonas de estudio cada uno.

\section{Bosque Tropical Siempre Verde Estacional Aciculifoliado Montano Inferior: El Bosque} Siempre Verde está conformado principalmente por especies del género Pinus (Mejía y House, 2002). Se trabajó en la zona de El Pericón, ubicado a 10 km sur de Yuscarán, entre 1100 a 1400 msnm, UTM 5160-70, 1537-38; y La Ciénaga, ubicado a $4 \mathrm{~km}$ al sur del Pericón, UTM 5150-60, 1534-35, entre 1000 a 1300 msnm. El clima de ambas zonas es húmedo y templado, con temperaturas mínimas de $15^{\circ} \mathrm{C}$ y máximas de $30^{\circ} \mathrm{C}$ (Argeñal, 2011).

Los principales usos de suelo encontrados en ambas zonas del Bosque Siempre Verde son bosque secundario, sistemas silvipastoriles, pastizalesmatorrales, cultivos de frijol y cultivos de maíz.

Arbustal Deciduo de Tierras Bajas: Es un ecosistema denominado comúnmente como bosque seco. Este se caracteriza principalmente por la caducidad de su vegetación durante la temporada seca, gran cantidad de arbustos espinosos y árboles de baja altura (Mejia y House, 2002). En el Arbustal Deciduo 
se trabajó en las zonas de El Rodeo, ubicado a $10 \mathrm{~km}$ sureste de Yuscarán, UTM 5235-40,1535-36 y Aguas Frías, UTM 5230-35, 1535-36. El clima de ambas zonas es seco y cálido, las temperaturas medias oscilan entre $17^{\circ} \mathrm{C}$ mínima y $40^{\circ} \mathrm{C}$ máxima (Argeñal, 2011). Los principales usos de suelo encontrados son bosque secundario, sistema silvipastoril, bosque bajo tala, pastizal y cultivos anuales.

Fase de campo y laboratorio: Las cuatro zonas de trabajo, El Pericón, La Ciénaga, El Rodeo y Aguas Frías, forman parte de la propuesta para Corredor Biológico La Unión. Cada zona se dividió en tres sitios de muestreo, según la estructura de la vegetación e intensidad en uso de suelo (Cuadro 1). En el Bosque Siempre Verde se catalogaron tres sitios: Bosque de Pino, Bosque Silvipastoril y Matorral (Figura 1); mientras que en el Arbustal Deciduo se asignaron las siguientes categorías: Bosque Deciduo, Bosque Deciduo Ralo y Pastizal (Figura 2).

En los sitios de muestreo se midió la temperatura ambiental, la temperatura del suelo y se determinó la vegetación predominante. En cada sitio de colecta se trazó un transecto lineal de $250 \mathrm{~m}$ de largo, con 5 trampas de caída distanciadas $50 \mathrm{~m}$ entre sí (Larsen y Forsyth, 2005). Las trampas estaban compuestas por dos vasos plásticos apilados, de $14 \mathrm{~cm}$ de alto y $10 \mathrm{~cm}$ de abertura, enterrados a ras del suelo y llenados con agua jabonosa hasta $1 / 4$ de su capacidad máxima.

Se insertaron sobre los vasos dos tiras de alambre de amarre galvanizado, utilizados para sostener el cebo envuelto con tela de nailon. El cebo consistió en $75 \mathrm{~g}$ de estiércol de vaca, el recurso más común y abundante de la zona.

Cada trampa fue cubierta con un techo trípode de aluminio para protegerla del sol y la lluvia. Las trampas se colocaron de julio a diciembre del 2012, monitoreándolas cada 48 horas posterior a su montaje, efectuando un total de 12 visitas por trampa, cada una con 576 horas acumuladas de actividad y un total de 2880 horas de colecta por sitio de muestreo.

El vaso superior se retiraba y se colocaban los escarabajos capturados en bolsas "Whirl Paks" ${ }^{\circledR}$ " con alcohol al 70\% para su conservación. En conjunto, se realizaron recolectas manuales mediante muestreos destructivos de las bostas encontradas en los transectos, con la finalidad de complementar la colecta de escarabajos con especies que las trampas no lograron capturar. Todos los sitios de muestreo fueron referenciados con un GPS Garmin eTrex ${ }^{\circledR}$ (Cuadro 1).

La determinación taxonómica de los individuos colectados fue con literatura especializada (Matthews, 1961; Jessop, 1985; Kohlmann, 1996; Kohlmann y Solís, 1997; Kohlmann y Solís, 2001; Solís y Kohlmann, 2002; Vaz-de-Mello et al., 2011; Edmonds y Zidek, 2012) y visitas a colecciones de referencia como el museo de Entomología UNAH y la Colección de Artrópodos de la Escuela Agrícola Panamericana, Zamorano, Honduras.

Análisis de datos: Se calculó la riqueza estimada de escarabajos por sitio de muestreo con el índice corregido de Chao (Chao, 1984), utilizando el programa SPADE (Chao y Shen, 2010). Con Ecosim 4.0 (Gotelli y Entsminger, 2001), se estimó el éxito de captura, calculando curvas de rarefacción basadas en el número de individuos, con 1000 randomizaciones.

Estas curvas de rarefacción permiten la comparación de diversos sitios de muestreo con distinto número de individuos (Gotelli y Colwell, 2011). Para el análisis de la diversidad alfa se empleó el índice de Diversidad de Shannon y Dominancia de Simpson, en base a los índices de diversidad alfa se crearon gráficas de barra, utilizando el programa PAST 3.01 (Hammer et al., 2001).

Se generó una matriz de similitud beta para cuantificar el porcentaje de igualdad entre las comunidades de cada sitio de muestreo. Se empleó el índice cuantitativo Bray Curtis, ya que es un coeficiente de similitud efectivo para datos crudos de abundancia (Legendre y Legendre, 1998).

Los escarabajos fueron catalogados en gremios según su manera de relocalizar el estiércol y tamaño corporal, utilizando los grupos propuestos por Cambefort (1991). Los gremios seleccionados fueron paracóprido grande (PG), paracóprido pequeño (PP), telocóprido grande (TG) y telocóprido pequeño (TP). Para encontrar la asociación y dependencia entre los gremios con el hábitat, se elaboró una tabla de contingencia, aplicando la prueba de independencia $\chi^{2}$. 
Cuadro 1. Factores abióticos y estructura vegetal en cada tipo de hábitat muestreado en El Pericón, La Ciénaga, El Rodeo y Aguas Frías de Yuscarán, departamento de El Paraíso, Honduras.

\begin{tabular}{|c|c|c|c|c|c|}
\hline $\begin{array}{l}\text { Uso de } \\
\text { Suelo }\end{array}$ & $\frac{\text { Temperatu }}{\text { Ambiental }}$ & $\frac{\text { ra }\left(\mathrm{C}^{\circ}\right)}{\text { Suelo }}$ & $\begin{array}{c}\text { Coordenada Inicial } \\
\text { y Final del } \\
\text { Transecto (UTM- } \\
\text { WGS 84) }\end{array}$ & $\begin{array}{l}\text { Altura } \\
\text { (msnm) }\end{array}$ & Descripción del Sitio \\
\hline $\begin{array}{l}\text { B.P El } \\
\text { Pericón }\end{array}$ & 21.6 & 19.2 & $\begin{array}{l}\text { I 515561,1536015 } \\
\text { F } 515677,1536117\end{array}$ & $\begin{array}{l}1200- \\
1350\end{array}$ & \multirow{2}{*}{$\begin{array}{l}\text { Bosque Secundario en estado natural de sucesión. No está } \\
\text { bajo ningún uso de suelo específico. Vegetación arbórea } \\
\text { compuesta principalmente por Pinus maximinoi. La altura } \\
\text { del dosel es de unos 17-18 m. Tiene un estrato arbustivo } \\
\text { ralo, conformado en su mayoría por Mimosa albida y } \\
\text { Buddleja americana y Calliandra grandiflora. }\end{array}$} \\
\hline $\begin{array}{l}\text { B.P La } \\
\text { Ciénaga }\end{array}$ & 24.7 & 22.0 & $\begin{array}{l}\text { I } 515377,1535873 \\
\text { F } 515519,1535794\end{array}$ & $\begin{array}{c}1350- \\
1420\end{array}$ & \\
\hline $\begin{array}{l}\text { B.S.P } \\
\text { El } \\
\text { Pericón }\end{array}$ & 27.8 & 27.4 & $\begin{array}{l}\text { I } 515670,1536062 \\
\text { F } 515831,1536001\end{array}$ & $\begin{array}{l}1200- \\
1290\end{array}$ & \multirow{2}{*}{$\begin{array}{l}\text { Bosque Ralo manejado bajo un sistema silvipastoril y } \\
\text { extracción artesanal de madera. En ambas áreas } \\
\text { disminuye el grosor, densidad y altura de los árboles (6-8 } \\
\mathrm{m} \text { de dosel), el estrato está conformado principalmente } \\
\text { por Pinus maximinoi y Quercus spp. El estrato arbustivo } \\
\text { aumenta en densidad y variedad de especies. }\end{array}$} \\
\hline $\begin{array}{l}\text { B.S.P } \\
\text { La } \\
\text { Ciénaga }\end{array}$ & 33.1 & 32.0 & $\begin{array}{l}\text { I } 515567,1535811 \\
\text { F } 515706,1535714\end{array}$ & $\begin{array}{c}1230- \\
1320\end{array}$ & \\
\hline $\begin{array}{l}\text { M. El } \\
\text { Pericón }\end{array}$ & 32.0 & 30.3 & $\begin{array}{l}\text { I } 515983,1536099 \\
\text { F } 516171,1536102\end{array}$ & $\begin{array}{l}1080- \\
1140\end{array}$ & \multirow{2}{*}{$\begin{array}{l}\text { Sitio usado como potrero ganadero y campos de siembra, } \\
\text { su vegetación principal son arbustos, Ludwigia peruviana } \\
\text { y Mimosa albida son las especies más abundantes. Posee } \\
\text { baja densidad y variedad de árboles, más especies } \\
\text { cultivadas como Manguifera indica, no obstante Pinus } \\
\text { maximinoi aún se encuentra más dispersa en el paisaje. }\end{array}$} \\
\hline $\begin{array}{l}\text { M. La } \\
\text { Ciénaga }\end{array}$ & 34.6 & 31.0 & $\begin{array}{l}\text { I } 515907,1535543 \\
\text { F } 516098,1535522\end{array}$ & $\begin{array}{c}1110- \\
1140\end{array}$ & \\
\hline $\begin{array}{l}\text { B.D El } \\
\text { Rodeo }\end{array}$ & 25.0 & 23.0 & $\begin{array}{l}\text { I 524141,1536101 } \\
\text { F 524293,1536184 }\end{array}$ & 450 & \multirow{2}{*}{$\begin{array}{l}\text { Bosque Secundario en sucesión natural, de dosel cerrado } \\
\text { y relativamente alto }( \pm 7 \mathrm{~m}) \text {, pero sus bordos están muy } \\
\text { intervenidos, los árboles dominantes son Bursera } \\
\text { simaruba, Diphysa americana, Bonellia nitida, Rehdera } \\
\text { trinervis y Allophylus psilospermus. El estrato arbustivo } \\
\text { se esparce desperdigado entre el arbóreo, las especies que } \\
\text { destacan son Acacia hindsii, Acacia farnesiana, } \\
\text { Exostema caribaeum y Mimosa tenuiflora. Estos bosques } \\
\text { poseen las temperaturas menos intensas. }\end{array}$} \\
\hline $\begin{array}{l}\text { B.D } \\
\text { Aguas } \\
\text { Frías }\end{array}$ & 25.4 & 23.8 & $\begin{array}{l}\text { I } 524693,1535406 \\
\text { F } 524879,1535424\end{array}$ & 450 & \\
\hline $\begin{array}{l}\text { B.D.R } \\
\text { El } \\
\text { Rodeo }\end{array}$ & 29.7 & 27.0 & $\begin{array}{l}\text { I } 524103,1536198 \\
\text { F } 524054,1536387\end{array}$ & 450 & \multirow{2}{*}{$\begin{array}{l}\text { Bosque Deciduo Ralo usado a veces como sitio para } \\
\text { extracción artesanal de madera y reposo del ganado. La } \\
\text { zona del dosel está muy dispersa, permitiendo mayor } \\
\text { entrada de luz lo que influye en las condiciones del suelo. } \\
\text { La variedad de árboles disminuye y aumenta el recambio } \\
\text { de especies, entre estas inciden Nopalea lutea, Senna } \\
\text { otomaria, Bonelia nitida y Exostema caribaeum. Los } \\
\text { arbustos más abundantes y frecuentes fueron Acacia, } \\
\text { Senna galegifolia y Acanthocereus tetragonus. }\end{array}$} \\
\hline $\begin{array}{l}\text { B.D.R. } \\
\text { Aguas } \\
\text { Frías }\end{array}$ & 27.0 & 25.6 & $\begin{array}{l}\text { I } 524369,1535451 \\
\text { F } 524229,1535337\end{array}$ & $\begin{array}{l}510- \\
570\end{array}$ & \\
\hline $\begin{array}{l}\text { P. El } \\
\text { Rodeo }\end{array}$ & 37.0 & 36.6 & $\begin{array}{l}\text { I } 523699,1536095 \\
\text { F } 523848,1535970\end{array}$ & $\begin{array}{l}450- \\
480\end{array}$ & \multirow{2}{*}{$\begin{array}{l}\text { Sitio muy intervenido, usado más como potrero en El } \\
\text { Rodeo y zona de cultivo/pastizal en Aguas Frías. Sitios } \\
\text { abiertos, con algunos individuos arbóreos/arbustivos de } \\
\text { manera ocasional, la vegetación está conformada } \\
\text { principalmente por gramíneas como Panicum maximun, } \\
\text { Cyperus sp., Sorghum bicolor y Andropogon sp. }\end{array}$} \\
\hline $\begin{array}{l}\text { P. } \\
\text { Aguas } \\
\text { Frías }\end{array}$ & 35.3 & 30.0 & $\begin{array}{l}\text { I } 524329,1535679 \\
\text { F } 524379,1535870\end{array}$ & 420 & \\
\hline
\end{tabular}



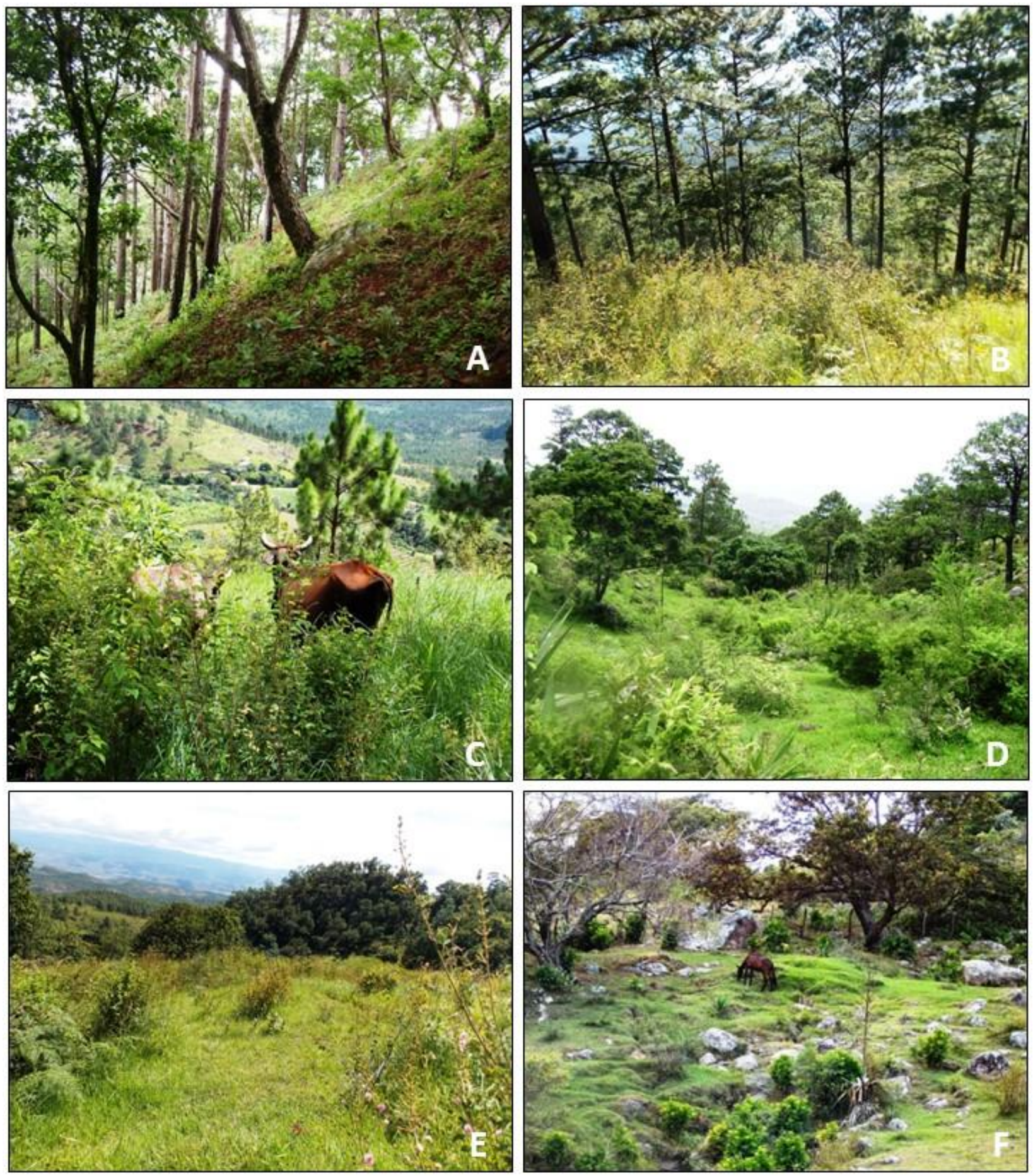

Figura 1. Sitios de muestreo en el Bosque Tropical Siempre Verde en Yuscarán, departamento de El Paraíso, Honduras. A) Bosque de Pino El Pericón, B) Bosque de Pino La Ciénaga, C) Bosque Silvipastoril El Pericón, D) Bosque Silvipastoril La Ciénaga, E) Matorral El Pericón y F) Matorral La Ciénaga. 

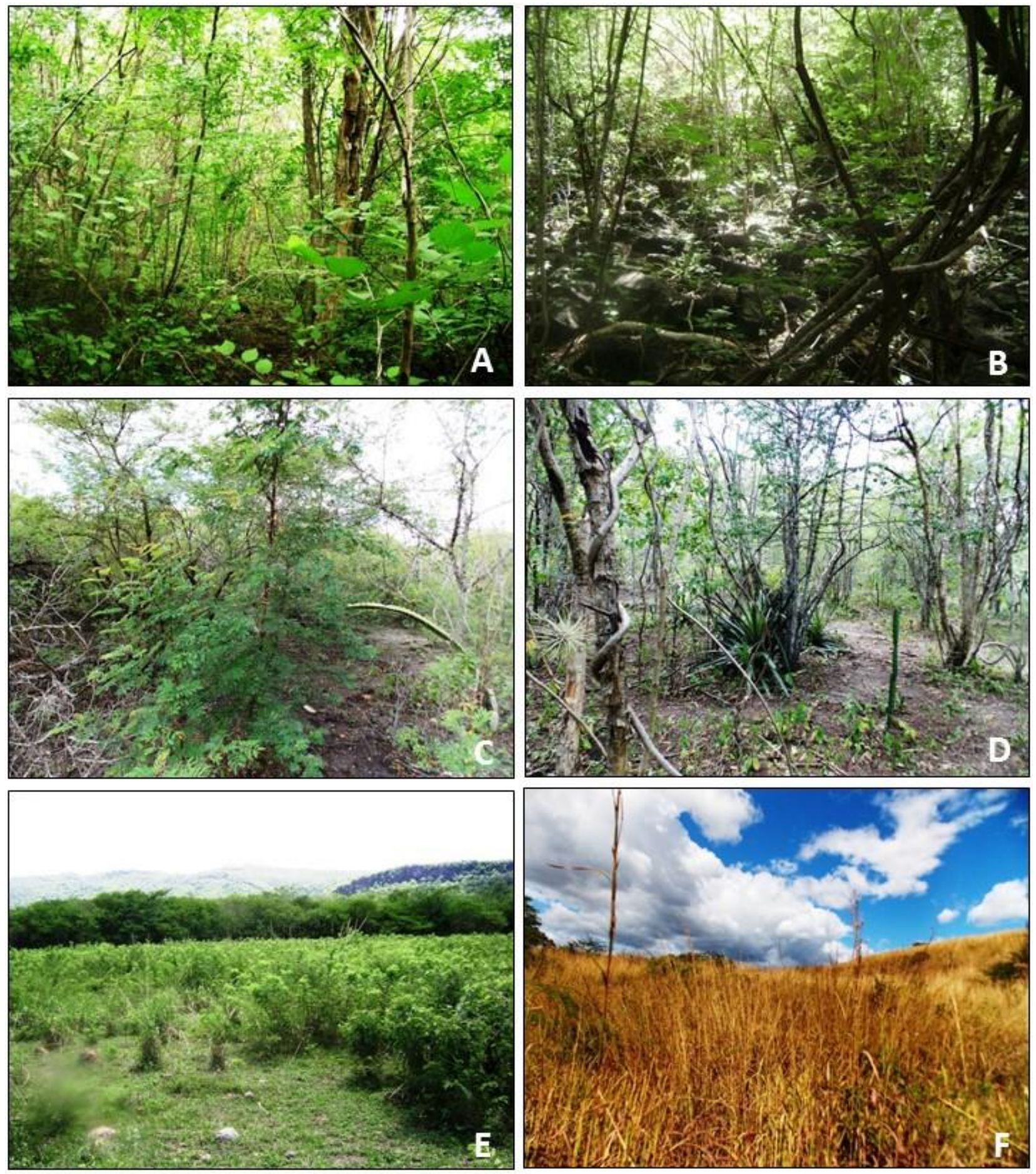

Figura 2. Sitios de muestreo en el Arbustal Deciduo de Tierras Bajas de Yuscarán, departamento de El Paraíso, Honduras. A) Bosque Deciduo El Rodeo, B) Bosque Deciduo Aguas Frías, C) Bosque Deciduo Ralo El Rodeo, D) Bosque Deciduo Ralo Aguas Frías, E) Pastizal El Rodeo y F) Pastizal Aguas Frías. 
Con el paquete estadístico R ( $\mathrm{R}$ core team, 2013) se análizó el escalamiento multidimensional no métrico (NMDS por sus siglas en inglés) basado en la abundancia de las especies y composición de los gremios. El presente método de ordenamiento es recomendado cuando los datos provienen de gradientes ambientales muy amplios con pocas especies en común (Ludwig y Reynolds, 1991).

Para verificar la efectividad del ordenamiento NMDS se usó el valor de estrés como prueba de bondad de ajuste, dicho valor es la diferencia entre las distancias medidas con las distancias observadas en el espacio de ordenamiento (Holland, 2008).

No se recomienda interpretar el ordenamiento NMDS con valores de estrés superiores a 0.3 , pues son resultados aleatorios con pobre ajuste de datos, mientras que los valores menores a 0.5 indican alta concordancia del ordenamiento con la naturaleza de los datos. A medida que el valor de estrés se acerque a 0.0, el ajuste de datos será más óptimo (Clarke, 1993).

\section{Resultados}

Riqueza de especies: Se capturaron 2,749 individuos de 9 tribus, 11 géneros y 21 especies (Cuadro 2). En El Pericón se colectaron 445 escarabajos de 10 especies. Las más abundantes fueron Dichotomius annae (35\%), Eurysternus magnus (23\%) y Copris lugubris (19\%). El $43 \%$ se capturó en el Bosque de Pino, $32 \%$ en el Bosque Silvipastoril y $19 \%$ en el Matorral (Cuadro 2). En La Ciénaga se colectaron 350 ejemplares de ocho especies y las más abundantes fueron $D$. annae $(61 \%)$, E. magnus (16\%) y C. lugubris (11\%). El 50\% de la colecta se capturó en el Bosque de Pino, el $27 \%$ en el Bosque Silvipastoril y 22\% en el Matorral (Cuadro 2).

En El Rodeo se colectaron 1006 escarabajos de 13 especies. Las especies más abundantes fueron Onthophagus landolti (40\%), Sisyphus mexicanus (17\%) y Onthophagus hoepfneri (17\%). El 40.8\% de la colecta se obtuvo en el Bosque Deciduo, 31.8\% en Bosque Deciduo Ralo y 27.3\% en Pastizal (Cuadro 2).

En Aguas Frías se colectaron 948 individuos de 14 especies. O. landolti (36\%), Ateuchus rodriguezi (21\%) y S. mexicanus (11\%) fueron las especies más abundantes. La incidencia de las demás especies se mantuvo por debajo del $10 \%$. El $42.6 \%$ de la colecta se capturó en el Bosque Deciduo, 37.1\% en el Bosque Deciduo Ralo y el $20.2 \%$ en el Pastizal (Cuadro 2).

Comparando la riqueza estimada con la riqueza observada (Sobs), los estimadores de riqueza infieren que se colectó más del $90 \%$ de especies pronosticadas para ambas áreas (Cuadro 3). Las curvas de rarefacción no alcanzaron su asíntota (Figura 3), lo que indica el potencial de cada hábitat por alcanzar más especies, en especial los sitios bajo menor intensidad de uso. No obstante, por su forma y pendiente, se considera que en los Pastizales (los sitios más degradados) se estuvo más cerca de alcanzar el número máximo de especies.

Diversidad alfa: Se estimaron los índices de diversidad alfa, comparando los resultados de ambos ecosistemas. El índice de diversidad en el Bosque Siempre Verde disminuyó a medida que la intensidad en el uso de suelo aumentaba (Figura 4A), simultáneamente el índice de Dominancia se incrementó (Figura 4B). Contrario al primer ecosistema, en las zonas del Arbustal Deciduo, el índice de diversidad fue mayor en los sitios bajo usos de suelo más intensos, como el Bosque Deciduo Ralo y el Pastizal (Figura 4A), mientras que el índice de Dominancia fue mayor en el Bosque Deciduo, el sitio bajo menor intensidad de uso (Figura 4B).

Diversidad beta: La matriz en base al índice cuantitativo de Bray Curtis indica que tan similares o diferentes son dos sitios según su estructura comunitaria. Un valor de 1.0 indica dos sitios con comunidades idénticas y 0.0 indica dos sitios con comunidades completamente diferentes. Según la matriz, el coeficiente de similitud entre los sitios de muestreo, en ambos ecosistemas, disminuye de manera progresiva al aumentar el grado de intervención del hábitat, siendo mayor el coeficiente de similitud entre los sitios bajo el mismo uso de suelo y menor entre usos de suelos diferentes (Cuadro 4A y B).

Estructura de los gremios: En el Bosque Siempre Verde se identificaron tres gremios: paracópridos grandes, paracópridos pequeños y telocópridos grandes (Cuadro 2). Hubo más diversidad de gremios en los sitios de menor intervención, como el Bosque 
de Pino y Bosque Silvipastoril, mientras que en el Matorral solo se encontraron paracópridos grandes (Figura 5A). La prueba de independencia $\chi^{2}$ probó que los gremios eran dependientes de los sitios de muestreo $(\mathrm{P}<0.0005)$.

En el Arbustal Deciduo se identificaron tres gremios, paracópridos grandes, paracópridos pequeños y telocópridos pequeños (Cuadro 2), los dos últimos fueron más frecuentes y abundantes en el Bosque Deciduo y Bosque Deciduo Ralo (Figura 5B). Según la prueba de independencia $\chi^{2}$ las frecuencias relativas de los gremios están relacionadas con el sitio de colecta. Los resultados fueron altamente significativos para ambas áreas $(\mathrm{P}<0.0005)$.

Cuadro 3. Riqueza de especies; B.P =Bosque de Pino, B.S.P. = Bosque Silvi Pastoril, M. = Matorral, B.D.= Bosque Deciduo, B.D.R. = Bosque Deciduo Ralo, $\mathrm{P}=$ Pastizal.

\begin{tabular}{lrcc}
\hline Sitio & Chao 1 & Sobs & \pm S.E \\
\hline B.P. El Pericón & 8.0 & 8 & 0.3 \\
B.S.P. El Perícón & 10.0 & 9 & 1.3 \\
M. El Pericón & 8.0 & 6 & 2.6 \\
B.P. La Ciénaga & 8.0 & 8 & 0.5 \\
B.S.P. La Ciénaga & 7.0 & 6 & 1.3 \\
M. La Ciénaga & 4.0 & 4 & 0.3 \\
B.D. El Rodeo & 12.0 & 11 & 1.3 \\
B.D.R. El Rodeo & 10.0 & 9 & 2.3 \\
P. El Rodeo & 8.0 & 8 & 0.0 \\
B.D. Aguas Frías & 11.0 & 10 & 2.3 \\
B.D.R. Aguas Frías & 14.0 & 13 & 1.4 \\
P. Aguas Frías & 12.0 & 12 & 0.5 \\
\hline
\end{tabular}

Análisis de ordenamiento: La gráfica de ordenamiento NMDS, basado en la abundancia de cada especie, con valor de estrés de 0.02, dividió los sitios de muestreo por ecosistema. Las distancias entre los distintos sitios separan las zonas más conservadas de las degradadas. La gráfica NMDS también muestra la afinidad de cada especie por un tipo de hábitat en particular (Figura 6A). El ordenamiento basado en la composición cuantitativa de los gremios (Figura 6B) mostró la preferencias de hábitat de los gremios en conjunto, con un valor de estrés de 0.01 . Los valores de estrés para ambas gráficas indican un ordenamiento de datos sumamente fiable, al ser valores menores de 0.1 (Clarke, 1993).

\section{Discusión}

Riqueza, abundancia y diversidad alfa: El número de individuos disminuyó al aumentar la intensidad del uso de suelo, independientemente del ecosistema (Cuadro 2). Lo mismo se encontró en investigaciones sobre el efecto del hábitat en las comunidades de escarabajos coprófagos (Howden y Nealis, 1975; Klein, 1989; Davis y Philips, 2005; Nichols et al., 2008). Por lo tanto, la disminución de su abundancia refleja la fuerte relación que tienen los escarabajos coprófagos con el estado de conservación del hábitat.

Los resultados infieren que paisajes intervenidos y abiertos generan condiciones ambientales y microclimáticas que sobrepasan el umbral de tolerancia de muchas especies sensibles. También, los hábitats abiertos pueden ocasionar condiciones adversas para la propagación y reproducción de los escarabajos, inclusive los más resistentes. Conforme a esta premisa, los Matorrales y Pastizales obtuvieron valores de abundancia muy bajos a pesar de estar compuestas casi en $100 \%$ por escarabajos generalistas y resistentes a hábitats antropogenizados (Cuadro 5).

En el Bosque Siempre Verde, las comunidades más ricas en especies, se congregaron en los sitios bajo menor intensidad de uso como el Bosque de Pino y Bosque Silvipastoril. Las comunidades de estos sitios estaban compuestas en su mayoría por especialistas forestales o escarabajos con mayor fidelidad por hábitats boscosos (Cuadro 5).

El alto porcentaje de especialistas forestales y sensibles a paisajes abiertos en los Bosques de Pino y Bosques Silvipastoriles puede ser producto del estatus de conservación de El Pericón y La Ciénaga, ya que forman parte de la zona de amortiguamiento de la Reserva Biológica de Yuscarán. Este factor contribuye a mantener más cobertura vegetal, indispensable para los escarabajos forestales (Halffter y Matthews, 1966).

Un bosque de dosel cerrado enriquece la calidad del estiércol, conservando su humedad por más tiempo y diversifica el alimento disponible para los escarabajos, como hongos, carroña y otra materia en descomposición (Gill, 1991). 
Cuadro 2. Categoría de gremio y número de individuos colectados por especie en cada tipo de hábitat dentro de El Pericón, La Ciénaga, El Rodeo y Aguas Frías. B.P=Bosque de Pino, B.S.P.= Bosque Silvi Pastoril, M.= Matorral, B.D.= Bosque Deciduo, B.D.R.= Bosque Deciduo Ralo, $\mathrm{P}=$ Pastizal; P.P=Paracóprido Pequeño, P.G= Paracóprido Grande, T.P= Telocóprido Pequeño, T.G= Telocóprido Grande; *N.R.= Nuevo Registro para Honduras.

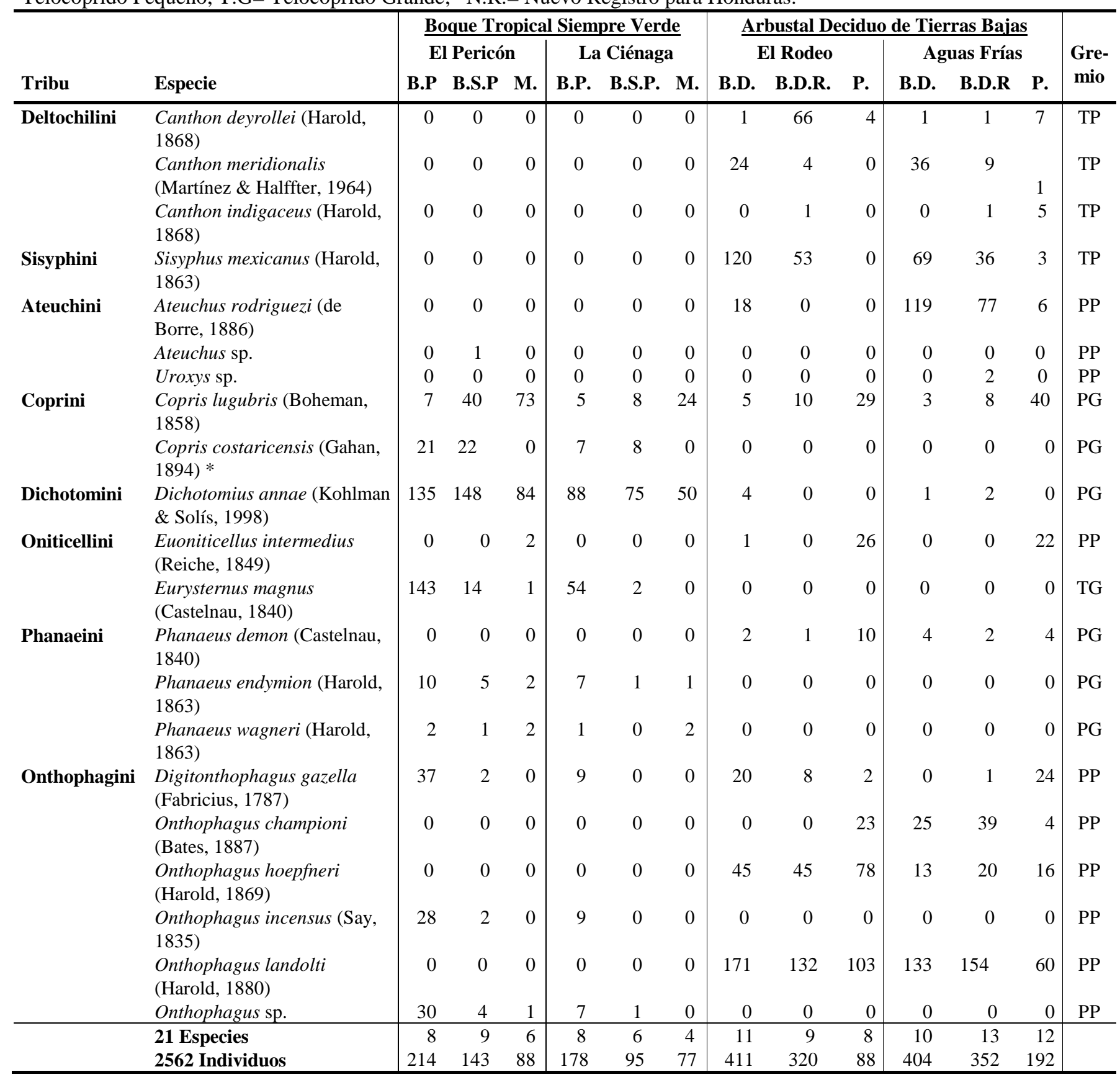


Contrario a los resultados de riqueza y diversidad, la curva de rarefacción del Matorral en El Pericón (Figura 3A), tiene una pendiente muy inclinada, infiriendo que puede ser potencialmente más rico en especies que el Bosque de Pino y Bosque Silvipastoril, no obstante, según Hulbert (1971), el resultado de la curva puede ser por la baja diversidad en El Matorral.

El estimador de riqueza y la pendiente de las curvas de rarefacción en las comunidades del Arbustal Deciduo, proponen que los sitios de muestreo se encuentran cerca de alcanzar su número máximo de especies (Cuadro 3; Figura 3D). Esto puede ser producto de las condiciones ambientales hostiles para los escarabajos coprófagos, ya que las comunidades estaban compuestas en su mayoría por especies generalistas (Cuadro 5), limitando así el número de especies que pueden establecerse en el ecosistema, como las sensibles a la perturbación, disminuyendo el potencial de riqueza en el área.

El índice de diversidad en El Rodeo, fue levemente mayor en los sitios más intervenidos, el Bosque Deciduo Ralo y el Pastizal; mientras que en Aguas Frías el índice de Diversidad fue notablemente mayor en el Pastizal, siendo este el hábitat más intervenido (Figura 4A), siendo las zonas más diversas, las más homogéneas en cuanto a la proporción relativa de cada especie.

Estas comunidades pueden acoplarse a la teoría de disturbio intermedio propuesta por Connel (1978), la cual propone que en ecosistemas muy degradados, el número máximo de diversidad e igualdad entre especies, se encuentra a niveles intermedios o moderadamente altos de perturbación, tal como el Arbustal Deciduo de Yuscarán (Figura 4A y B), producto de especies turistas y generalistas. Otros autores ya han demostrado que la riqueza y diversidad puede ser mayor en hábitats más degradados (Halffter et al., 1992; Louzada et al., 2010; Giraldo et al., 2011, Braga et al., 2013).

Diversidad beta: De acuerdo a la matriz de similitud (Cuadro 4), las comunidades de escarabajos coprófagos se diferenciaban más a medida que la intensidad en el uso del suelo aumentaba y disminuía la cobertura vegetal, mostrando como la intensidad de perturbación alteraba las comunidades de forma gradual. No obstante, los porcentajes de similitud entre las comunidades del Bosque Deciduo eran relativamente mayores a las obtenidas en el Bosque Siempre Verde (Cuadro 4), con lo cual se puede inferir que en el ecosistema seco no hubo grandes diferencias entre las comunidades de hábitats boscosos y la de los hábitats abiertos, a pesar de las distintas condiciones microclimáticas (Cuadro 1). Por lo tanto, basado en los resultados de similitud (Cuadro 4) es factible considerar que las comunidades forestales en el ecosistema seco están en proceso de homogenización faunística.

Estructura de los gremios: La prueba de independencia comprobó que los porcentajes relativos de cada gremio encontrado fueron dependientes del nivel de intervención humana. A medida que la intervención aumentó, la estructura y composición de los gremios tendía a simplificarse (Figura 5). Casos similares han reportado Estrada y Coates-Estrada (2002), Escobar (2004) y Navarrete y Halffter (2008). Por lo tanto, la perturbación humana también incide en las dinámicas ecológicas de los escarabajos coprófagos al simplificar la estructura de sus gremios.

La pérdida de la variabilidad ecológica de los gremios puede repercutir negativamente en la funcionalidad y sostenibilidad de un ecosistema (Andressen, 2003), al simplificar la compleja estructura trófica en un hábitat, disminuyendo el alcance de los servicios ecológicos provistos por Scarabaeinae.

Análisis de ordenamiento: El análisis de ordenamiento NMDS separa las especies por preferencia de hábitat. En el Bosque Siempre Verde, E. magnus, Onthophagus incensus y Onthophagus sp. son escarabajos con alta fidelidad por paisajes boscosos (Figura 5A). Debido a la distintiva forma de E. magnus para elaborar cámaras de crianza, necesita un suelo muy húmedo y suave (Halffter, 1997), tal como se puede encontrar en el Bosque de Pino. 

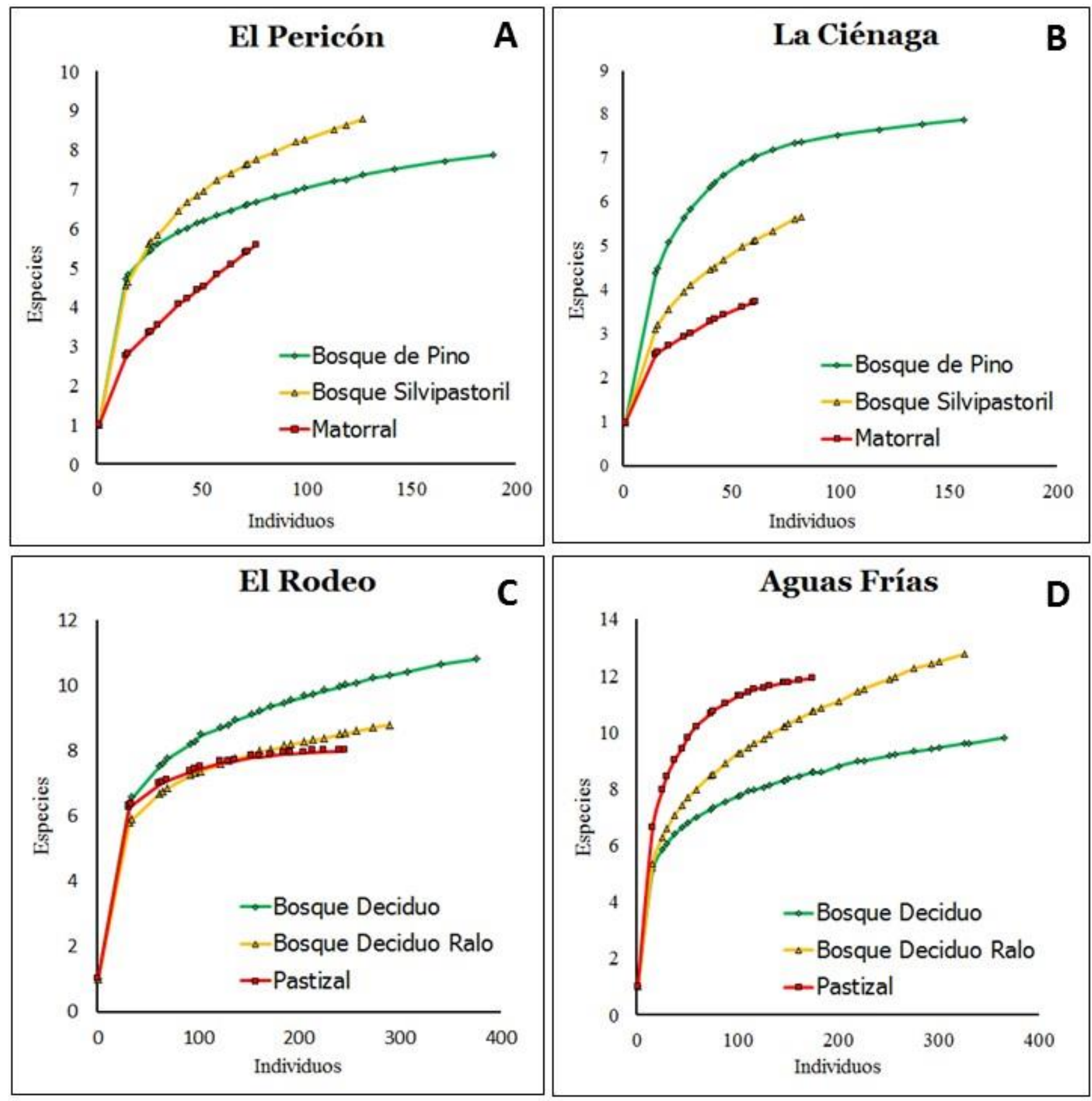

Figura 3. Curvas de Rarefacción basada en el número de individuos. A) El Pericón, B) Ciénaga, C) El Rodeo y D) Aguas Frías, comunidades de Yuscarán, departamento de El Paraíso, Honduras. 

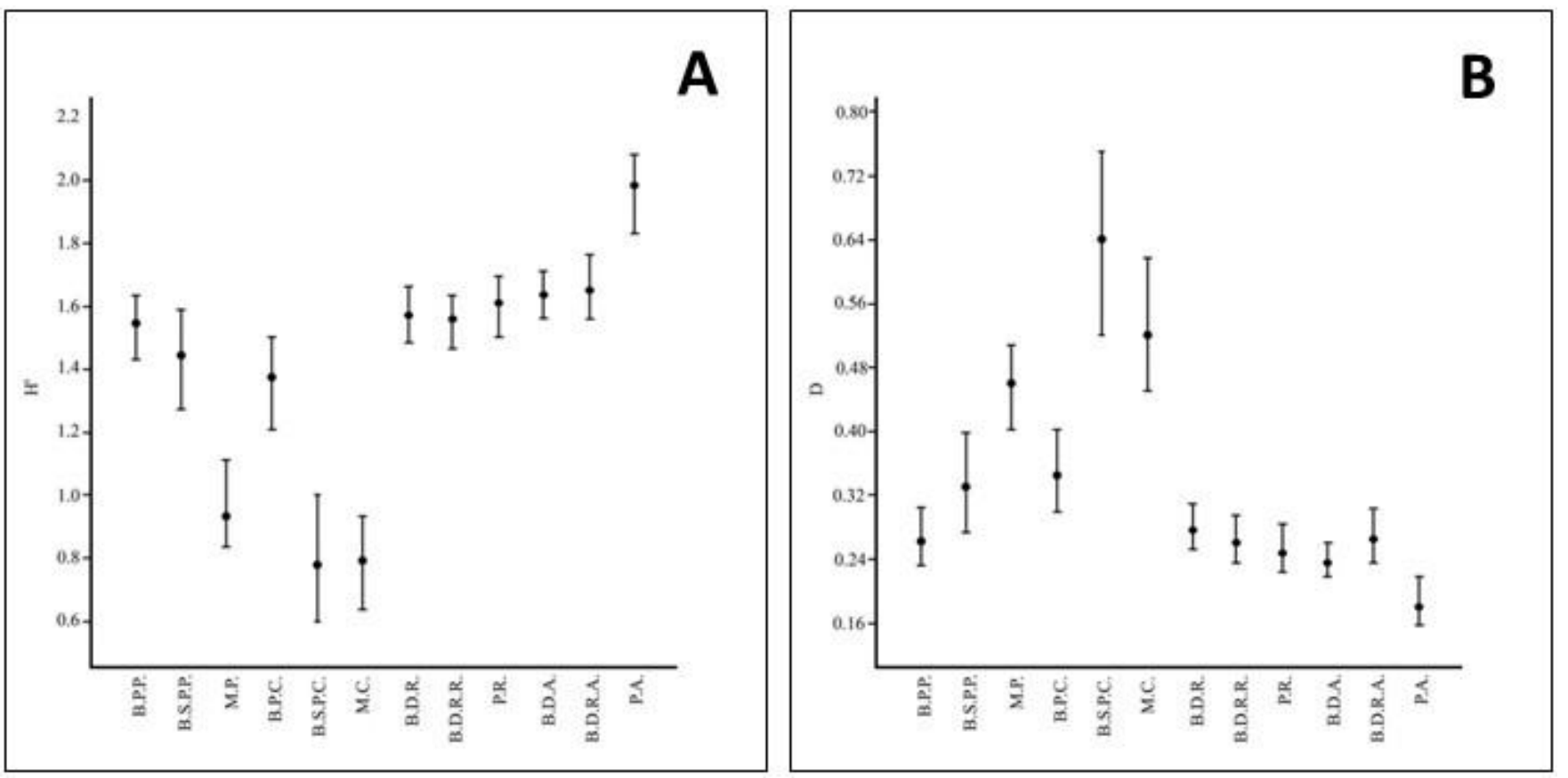

Figura 4. A) Diversidad de Shannon $\left(\mathrm{H}^{\prime}\right) \pm$ S.E; B) Dominancia de Simpson (D) \pm S.E. B.P.P $=$ Bosque de Pino Pericón, B.S.P.P= Bosque Silvipastoril Pericón, M.P= Matorral Pericón, B.P.C = Bosque de Pino Ciénaga, B.S.P.C = Bosque Silvipastoril Ciénaga, M.C = Matorral Ciénaga, B.D.R= Bosque Deciduo Rodeo, B.D.R.R= Bosque Deciduo Ralo Rodeo, P.R= Pastizal Rodeo, B.D.A= Bosque Deciduo Aguas Frías, B.D.R.A= Bosque Deciduo Ralo Aguas Frías, P.A= Pastizal Aguas Frías.

Cuadro 4. Matriz de comparaciones pareadas de sitios bajo distinta intensidad de uso en base al índice de Similitud-Bray Curtis, A) Bosque Siempre Verde -El Pericón y La Ciénaga; B) Arbustal Deciduo - El Rodeo y Aguas Frías; B.P.P= Bosque de Pino Pericón, B.S.P.P= Bosque Silvipastoril Pericón, M.P= Matorral Pericón, B.P.C = Bosque de Pino Ciénaga, B.S.P.C = Bosque Silvipastoril Ciénaga, M.C= Matorral Ciénaga, B.D.R= Bosque Deciduo Rodeo, B.D.R.R= Bosque Deciduo Ralo Rodeo, P.R= Pastizal Rodeo, B.D.A= Bosque Deciduo Aguas Frías, B.D.R.A= Bosque Deciduo Ralo Aguas Frías, P.A= Pastizal Aguas Frías.

A
\begin{tabular}{|l|cccccc|}
\hline & BPP & BSPP & MP & BPC & BSPC & MC \\
\hline \hline BPP & $\mathbf{1 . 0}$ & 0.476 & 0.258 & 0.663 & 0.395 & 0.351 \\
BSPP & - & $\mathbf{1 . 0}$ & 0.597 & 0.673 & 0.782 & 0.691 \\
MP & - & - & $\mathbf{1 . 0}$ & 0.316 & 0.492 & 0.715 \\
BPC & - & - & - & $\mathbf{1 . 0}$ & 0.667 & 0.447 \\
BSPC & - & - & - & - & $\mathbf{1 . 0}$ & 0.686 \\
MC & - & - & - & - & - & $\mathbf{1 . 0}$ \\
\hline
\end{tabular}

B
\begin{tabular}{|l|cccccc|}
\hline \multicolumn{1}{|l}{} & BDR & BDRR & PR & BDA & BDRA & PA \\
\hline \hline BDR & $\mathbf{1 . 0}$ & 0.681 & 0.464 & 0.697 & 0.700 & 0.328 \\
BDRR & - & $\mathbf{1 . 0}$ & 0.555 & 0.594 & 0.628 & 0.402 \\
PR & - & - & $\mathbf{1 . 0}$ & 0.371 & 0.437 & 0.685 \\
BDA & - & - & - & $\mathbf{1 . 0}$ & 0.794 & 0.319 \\
BDRA & - & - & - & - & $\mathbf{1 . 0}$ & 0.379 \\
PA & - & - & - & - & - & $\mathbf{1 . 0}$ \\
\hline
\end{tabular}




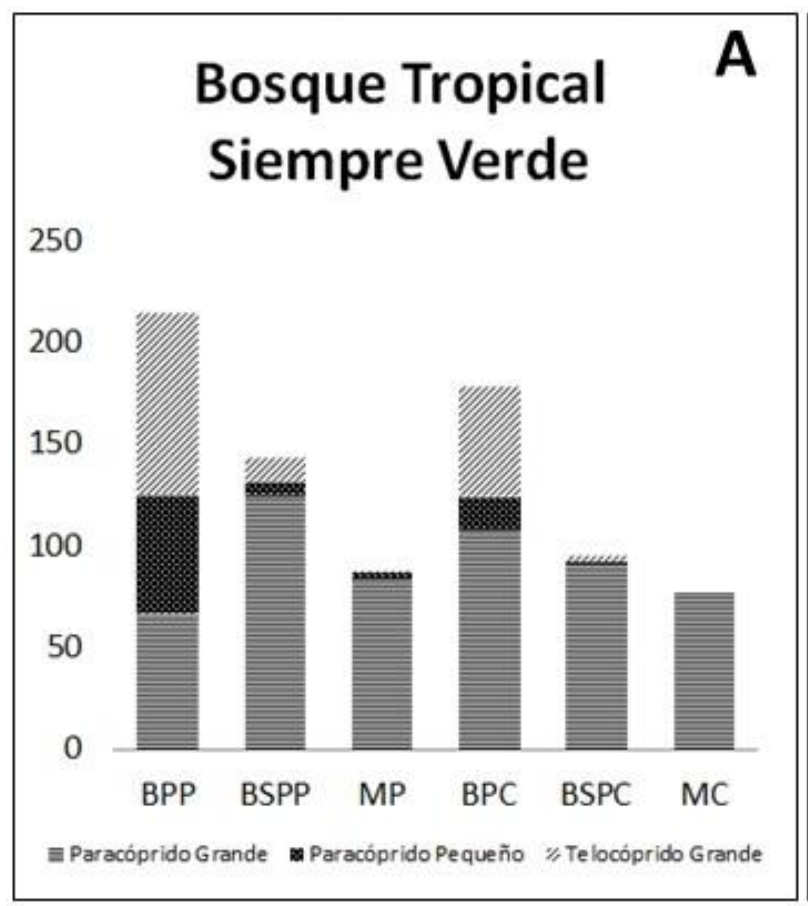

$\chi^{2}=228.923 ;$ G.L $=4 ; P<0.000001$

\section{Arbustal Deciduo de Tierras Bajas}

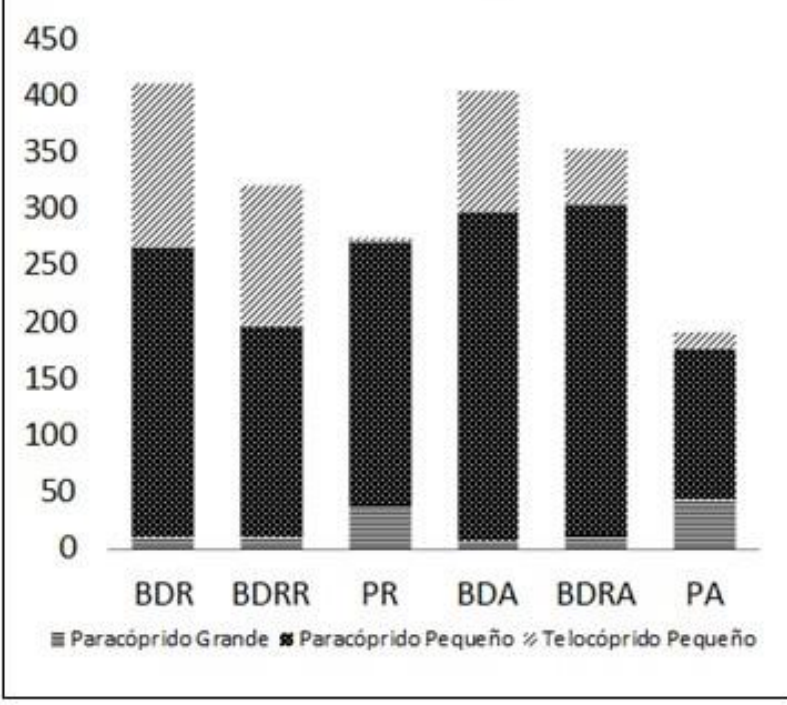

$\chi^{2}=225.847 ;$ G.L $=4 ;$ P $<0.000001$

Figura 5. Contribución de cada gremio a la abundancia total por sitio de colecta, A) Bosque Tropical Siempre Verde, B) Arbustal Deciduo de Tierras Baja; B.P.P= Bosque de Pino Pericón, B.S.P.P= Bosque Silvipastoril Pericón, M.P= Matorral Pericón, B.P.C= Bosque de Pino Ciénaga, B.S.P.C= Bosque Silvipastoril Ciénaga, M.C= Matorral Ciénaga, B.D.R= Bosque Deciduo Rodeo, B.D.R.R= Bosque Deciduo Ralo Rodeo, P.R= Pastizal Rodeo, B.D.A= Bosque Deciduo Aguas Frías, B.D.R.A= Bosque Deciduo Ralo Aguas Frías, P.A= Pastizal Aguas Frías.

Además, producto de la cercanía de sus nidos con la superficie del suelo, pierden humedad rápidamente en paisajes abiertos y más calientes como los Matorrales (Cuadro 1), aumentando la tasa de mortalidad de sus larvas (Fincher, 1973). Un caso similar ocurre con O. incensus y Onthophagus sp. que por su pequeño tamaño, son muy ineficaces excavando cámaras de crianza en suelos endurecidos y secos.

En el Arbustal Deciduo, A. rodriguezi, $S$. mexicanus y Canthon meridionalis mostraban mayor preferencia por hábitats boscosos (Figura 6A). Sisyphus mexicanus y C. meridionalis (Cuadro 2) estaban acondicionadas por las características abióticas del entorno y sus limitaciones físicas. Dichas limitaciones son producto de sus adaptaciones evolutivas para rodar el estiércol y menor tamaño, las cuales reducen su fuerza de excavación (Hanski y Camberfort, 1991). Por lo tanto, a estas especies posiblemente se les dificultaba excavar en suelos endurecidos y secos por la fuerte radiación solar y mayores temperaturas de hábitats abiertos como el Bosque Deciduo Ralo y Pastizal (Cuadro 1), siendo excluidos de estas zonas.

Contrario a $S$. mexicanus y Canthon meridionalis, C. deyrollei y Canthon indigaceus mostraron preferencias por hábitats abiertos y semiabiertos (Figura 6A), catalogándoles como escarabajos heliofílicos. Algunas especies del género Canthon 
están acondicionadas a temperaturas externas, pues al calentarse alcanzan mayor movilidad, más capacidad para construir esferas de crianza y más potencia de excavación (Bartholomew y Heinrich, 1978). Por tal motivo, a $C$. deyrollei y $C$. indigaceus se les encuentra ligadas a bordes forestales y paisajes abiertos, donde la intensidad lumínica y calorífica es mayor (Cuadro 1). Digitonthophagus gazella y Euoniticellus intermedius, también escarabajos heliofílicos, se sesgaron fuertemente hacia los pastizales (Figura 6A). Considerándoles como especies indicadoras de paisajes abiertos y muy degradados (Escobar, 2007). Las otras especies en el análisis de ordenamiento (Figura 6a), no mostraron una marcada presencia por un hábitat, permutando entre paisajes boscosos $\mathrm{y}$ abiertos, en especial C. lugubris.

C. lugubris se encontró en los dos ecosistemas y todos los sitios de muestreo (Figura 6A), considerándole como una especie altamente sinantropogénica y factiblemente el escarabajo más resistente a la degradación humanas. El hábito nocturno de $C$. lugubris le permitió establecerse exitosamente en todos los usos de suelo, incluso los abiertos y altamente intervenidos, pues al restringir sus actividades durante la noche, mitiga los efectos negativos de elevar su temperatura corporal lo suficiente para producir un choque térmico durante el vuelo (Verdú et al., 2006).

Según la proporción relativa de los gremios (Figura 5) y análisis de ordenamiento (Figura 6B), los telocópridos grandes del Bosque Siempre Verde y los telocópridos pequeños del Arbustal Deciduo fueron los gremios de mayor sensibilidad a la perturbación del hábitat, al distanciarse más de los usos de suelo más intervenidos. Por lo tanto, la degradación del ambiente en Yuscarán implica un severo cambio en la estructura de las comunidades, alterando negativamente no solo la diversidad de especies, sino también la estructura trófica de sus gremios.

\section{Conclusiones}

La comunidad de escarabajos coprófagos (Scarabaeidae: Scarabaeinae) en el Bosque Siempre Verde es alterada y modificada negativamente por el grado de intervención humana. Sin embargo, aún conserva una buena tasa de especies forestales, por lo tanto, en este ecosistema es prioritario la manutención de los bosques que contribuyen a generar condiciones idóneas para la perpetuación de muchas especies sensibles e importantes.

En el Arbustal Deciduo, la comunidad de escarabajos coprófagos está compuesta por especies más resistentes a la intervención humana y de hábitos generalistas, relegando y reduciendo la cantidad de especialistas forestales. Factiblemente, la composición y estructura de las comunidades boscosas del Arbustal Deciduo están en procesos de homogenización faunística, beneficiando el establecimiento de escarabajos más resistentes a hábitats con menor cobertura vegetal.

La intervención humana influye en la estructura y organización de los gremios de ambos ecosistemas, lo que disminuye su variedad y homogeneidad, disminuyendo el alcance de servicios ambientales prestados por Scarabaeinae.

El estudio es un reflejo realista y preocupante del detrimento actual de los ecosistemas secos en Yuscarán, requiriendo urgentemente medidas para su protección, considerando el panorama de la situación actual de Yuscarán como no positivo. No obstante, con el estudio también se intuye el efecto benéfico de la protección estatal hacia los ecosistemas forestales, necesitando aumentar los esfuerzos por mantener y proteger el bosque, especialmente los deciduos, así se conservará la biodiversidad y recursos que tanto dependen las comunidades rurales y pobres.

\section{Agradecimientos}

Se le agradece al Proyecto Ecosistemas y Fundación Yuscarán por financiar parte de esta investigación. A la familia Castellanos y Montenegro por recibirme tan cálidamente en sus hogares durante el desarrollo del trabajo. Igualmente, se le agradece a Juan Carlos Castellanos y Antonio Montenegro por su apoyo en el campo, también se le agradece al Museo de Entomología de la Escuela Agrícola Panamericana, Honduras, por su apoyo durante la identificación y en especial al Dr. Oliver Schlein por sus aportaciones y consejos para el mejoramiento del trabajo. 

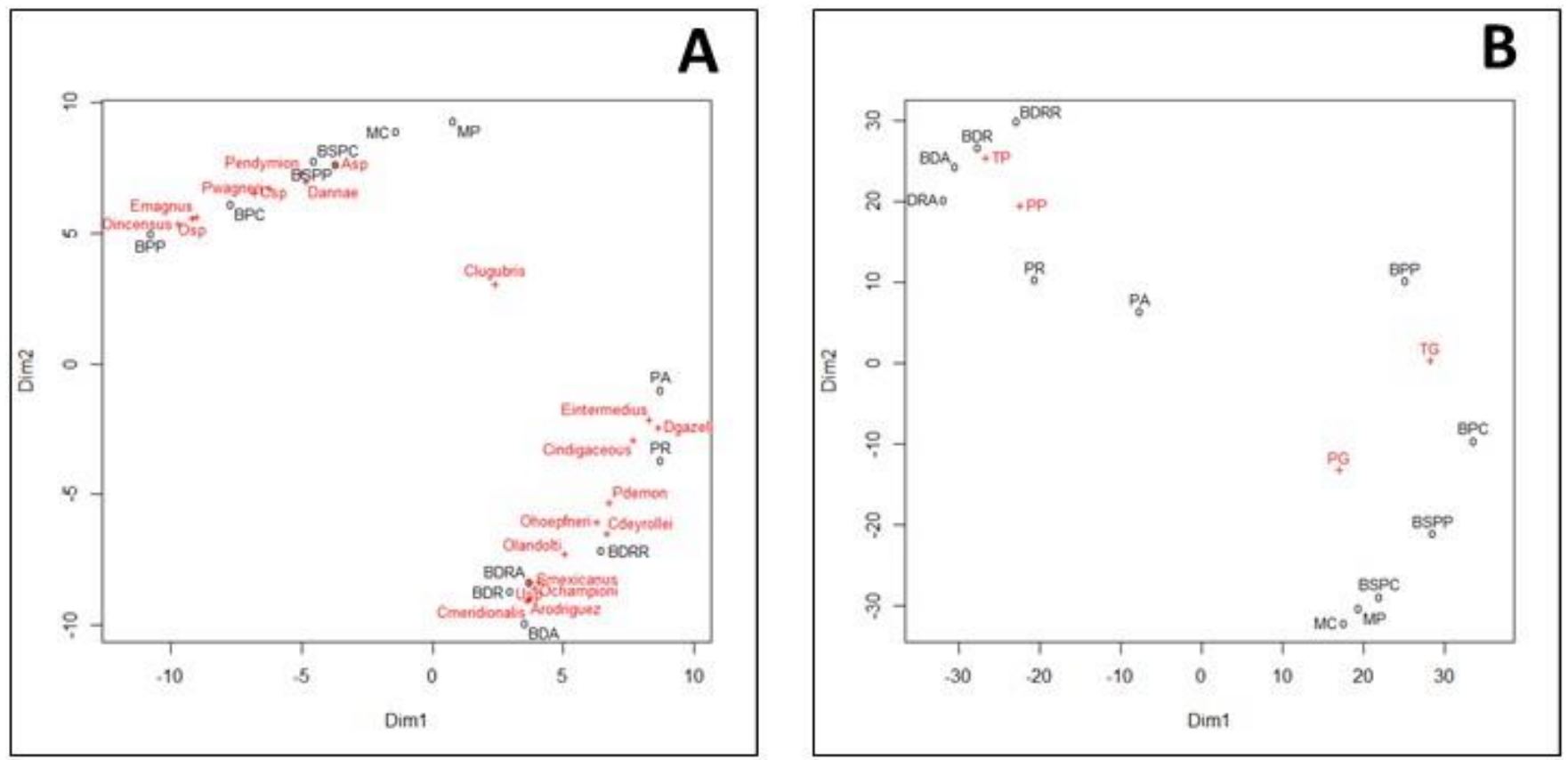

Figura 6. A) NMDS por abundancia de especies; B) NMDS por composición de gremios; Emagnus= Eurysternus magnus, Oincensus= Onthophagus incensus, Osp= Onthophagus sp., Ohoepfneri= Onthophagus hoepfneri, Olandolti= Onthophagus landolti, Ochampioni= Onthophagus championi, Arodriguezi=Ateuchus rodriguezi, Asp.= Ateuchus sp., Usp.= Uroxys sp., Pwagneri= Phanaeus wagneri; Pendymion= Phanaeus endymion, Pdemon=Phanaeus demon, Clugubris $=$ Copris lugubris, Csp. $=$ Copris. $\mathrm{sp}$, Danne $=$ Dichotomous annae, Eintermedius=Euoniticellus intermedius, Dgazella $=$ Digitonthophagus gazella , Cindigaceus= Canthon indigaceus, Cdeyrollei=Canthon deyrollei, Cmeridionalis $=$ Canthon meridionalis, Smexicanus $=$ Sisyphus mexicanus; $\mathrm{TP}=$ Telocópridos Pequeños, $\mathrm{TG}=$ Telocópridos Grandes, $\mathrm{PP}=$ Paracópridos Pequeños, $\mathrm{PG}=$ Paracópridos Grandes.

\section{Literatura Citada}

Andresen, E. 2003. Effect of forest fragmentation on dung beetle communities and functional consequences for plant regeneration. Ecography 26(1):87-97.

Argeñal, L. 2011. Precipitación y Temperatura media anual de Corredor Biológico La Union. Güinope, Yuscarán y Oropolí El Paraíso [Mapa]. Escala no vista. ICF, SAG. Proyecto Ecosistemas. Diciembre, 2011. ARCGIS: [GIS]. Tegucigalpa, Honduras: Proyecto Ecosistemas.

Arnaudin, M. 2012. Benefits of dung beetles (Coleoptera: Scarabaeidae) on nutrient cycling and forage growth in alpaca pastures. Tesis de Maestría. Virginia Polytechnic Institute and State University, Blacksburg, VA. 97 p.
Bang, H., J. Lee, O. Kwon, Y. Na, Y. Jang y W. Kim. 2005. Effects of paracoprid dung beetles (Coleoptera: Scarabaeidae) on the growth of pasture herbage and on the underlying soil. Applied Soil Ecology 29(2):165-171.

Barbier, E. y J. Bishop. 1995. Economic and social values affecting soil and water conservation in developing countries. Journal of Soil and Water Conservation 50(2):133-135.

Barragán, F., C. Moreno, F. Escobar, G. Halffter y D. Navarrete. 2011. Negative impacts of human land use on dung beetle functional diversity. PLos One 6(2): e17976.

Barrance, A., K. Schreckenberg y K. Gordon. 2009. Conservación mediante el uso: lecciones aprendidas en el bosque seco tropical mesoamericano. ODI. Londres. 158 p. 
Bartholomew, G. y B. Heinrich. 1978. Endothermy in African dung beetles during flight, ball making, and ball rolling. The Journal of Experimental Biology 73(1):6583.

Bertone, M., J. Green, S. Washburn y M. Poore. 2006. The contribution of tunneling dung beetles to pasture soil nutrition. Forage and Grazinglands. Documento en Línea. DOI 10.1094/FG-2006-0711-02-RS.

Braga R., V. Korasaki, E. Andresen y J. Louzada. 2013. Dung beetle community and functions along a habitatdisturbance gradient in the Amazon: A rapid assessment of ecological functions associated to biodiversity. PLoS ONE8(2): e57786.doi:10.1371/journal.pone.0057786.
Brown, J., C. Sholtz, J. Janeau, S. Grellier, y P. Podwojewski. 2010. Dung beetles (Coleoptera: Scarabaeidae) can improve soil hydrological properties. Applied Soil Ecology 46(1):9-16.

Cambefort, Y. 1991. Biogeography and evolution. In Hanski, I e Y. Camberfort (eds.). Dung beetle ecology. Princeton University Press. Princeton. Nueva Jersey. $p$ 51-67.

CELADE, UNFPA. 2005. Dinámica demográfica y desarrollo en América Latina y el Caribe. Serie Población y Desarrollo. 54 p.

Chao, A. 1984. Nonparametric estimation of the number of classes in a population. Scandinavian Journal of Statistics 11(4): 265-270.

Cuadro 5. Porcentaje relativo de captura por sitio de muestreo. Bosque Siempre Verde - Porcentaje de especies con mayor preferencias hacia hábitats boscosos (BP y BSP) vs. Porcentaje de especies con mayor preferencia hacia hábitats abiertos (BSP y M) = 70:30; Arbustal Deciduo - Porcentaje de especies con mayor preferencias hacia hábitats boscosos (BD y BDR) vs. Porcentaje de especies con mayor preferencia hacia hábitats abiertos $(\mathrm{BDR}$ y $\mathrm{P})=\mathbf{5 0 : 5 0}$; $\mathrm{B} . \mathrm{P}=$ Bosque de Pino, $\mathrm{BSP}=$ Bosque Silvi Pastoril, $\mathrm{M}=$ Matorral, $\mathrm{BD}=\mathrm{Bosque}$ Deciduo, $\mathrm{BDR}=$ Bosque Deciduo Ralo, $\mathrm{P}=$ Pastizal; $\mathrm{PH}=$ Preferencia de Hábitat; $\mathrm{H}=$ Heliofilico, $\mathrm{E}=$ Especialista, $\mathrm{G}=$ Generalista, $\mathrm{GF}=$ Generalista Forestal; $\varnothing=$ colectados una sola vez.

\begin{tabular}{|c|c|c|c|c|c|c|c|}
\hline Especie & $\mathbf{B P}$ & $\overline{\text { BSP }}$ & $\mathbf{M}$ & BD & $\overline{\text { BDR }}$ & $\mathbf{P}$ & $\overline{\mathbf{P H}}$ \\
\hline Canthon deyrollei (Harold, 1868) & 0 & 0 & 0 & 2 & 84 & 14 & $\mathbf{H}$ \\
\hline Canthon meridionalis (Martínez \& Halffter, 1964) & 0 & 0 & 0 & 81 & 18 & 1 & $\mathbf{E}$ \\
\hline Canthon indigaceus (Harold, 1868) & 0 & 0 & 0 & 0 & 29 & 71 & $\mathbf{H}$ \\
\hline Sisyphus mexicanus (Harold, 1863) & 0 & 0 & 0 & 67 & 32 & 1 & $\mathbf{E}$ \\
\hline Ateuchus rodriguezi (de Borre, 1886) & 0 & 0 & 0 & 62 & 35 & 3 & GF \\
\hline Ateuchus sp. & 0 & 100 & 0 & 0 & 0 & 0 & $\emptyset$ \\
\hline Uroxys sp. & 0 & 0 & 0 & 0 & 100 & 0 & $\emptyset$ \\
\hline Copris lugubris (Boheman, 1858) & 6 & 33 & 61 & 8 & 19 & 73 & $\mathbf{G}$ \\
\hline Copris costaricensis (Gahan, 1894) & 49 & 51 & 0 & 0 & 0 & 0 & GF \\
\hline Dichotomius annae (Kohlman \& Solís, 1998) & 37 & 40 & 23 & 71 & 29 & 0 & GF \\
\hline Euoniticellus intermedius (Reiche, 1849) & 0 & 0 & 100 & 2 & 0 & 98 & $\mathbf{H}$ \\
\hline Eurysternus magnus (Castelnau, 1840) & 90 & 9 & 1 & 0 & 0 & 0 & $\mathbf{E}$ \\
\hline Phanaeus demon (Castelnau, 1840) & 0 & 0 & 0 & 29 & 13 & 61 & $\mathbf{G}$ \\
\hline Phanaeus endymion (Harold, 1863) & 59 & 29 & 12 & 0 & 0 & 0 & GF \\
\hline Phanaeus wagneri (Harold, 1863) & 40 & 20 & 40 & 0 & 0 & 0 & $\mathbf{G}$ \\
\hline Digitonthophagus gazella (Fabricius, 1787) & 0 & 0 & 0 & 0 & 2 & 98 & $\mathbf{H}$ \\
\hline Onthophagus championi (Bates, 1887) & 0 & 0 & 0 & 46 & 48 & 6 & GF \\
\hline Onthophagus hoepfneri (Harold, 1869) & 0 & 0 & 0 & 27 & 30 & 43 & $\mathbf{G}$ \\
\hline Onthophagus incensus (Say, 1835) & 95 & 5 & 0 & 0 & 0 & 0 & $\mathbf{E}$ \\
\hline Onthophagus landolti (Harold, 1880) & 0 & 0 & 0 & 40 & 38 & 22 & GF \\
\hline Onthophagus sp. & 86 & 12 & 2 & 0 & 0 & 0 & $\mathbf{E}$ \\
\hline
\end{tabular}


Chao, A. y T. Shen. 2010. Program SPADE (Species Prediction And Diversity Estimation). Program and User's Guide. Recuperado de: http://chao.stat.nthu.edu.tw.

Clarke, K. 1993. Non-parametric multivariate analyses of changes in community structure. Australian Journal of Ecology 18(1): 117-143.

Connel, J. 1978. Diversity of tropical rain forest and coral reefs. Science 199(4345):1302-1310.

Davis, A. y K. Philips. 2005. Effect of deforestation on a southwest Ghana dung beetle assemblage (Coleoptera: Scarabaeidae) at the periphery of Anaska conservartion area. Enviromental Entomology 34(5):1081-1088.

Doube, B., A. Macqueen, T. Ridsdill-Smith y T. Weir. 1991. Native and introduced dung beetles in Australia. In Hanski, I. y Y. Camberfort (eds.). Dung beetle ecology. Princeton University Press. Princeton, Nueva Jersey. p. 255-278.

Edmonds, W. y J. Zídek. 2012. Taxonomy of Phanaeus revisited: revised keys to and comments on species of the new world dung beetle genus Phanaeus MacLeay, 1819 (Coleoptera: Scarabaeidae: Scarabaeinae: Phanaeini). Insecta Mundi 0274: 1-108.

Eitzinger, A., K. Sonder, A. Schmidt y K. Hagan. 2012. Tortillas on the roaster summary report: Central American maize-bean system and the changing climate. Catholic Relief Services, CIAT, CIMMYT y Howard G. Buffett Foundation. Baltimore. 17 p.

Escobar, F. 2004. Diversity and composition of dung beetle (Scarabaeinae) assemblages in a heterogeneous Andean landscape. Tropical Zoology 17(1):123-136.

Estrada, A. y R. Coates-Estrada. 1991. Howler monkeys (Alouatta palliata), dung beetles (Scarabaeidae) and seed dispersal: ecological interactions in the tropical rain forest of Los Tuxtlas, Mexico. Journal of Tropical Ecology 7(4):459-474.

Estrada, A. y R. Coates-Estrada. 2002. Dung beetles in continuous forest, forest fragments and in an agricultural mosaic habitat island at Los Tuxtlas, Mexico. Biodiversity \& Conservation 11(11): 1903-1918.

Fincher, G. 1973. Nidification and reproduction of Phanaeus spp. in three textural classes of soil (Coleoptera: Scarabaeidae). The Coleopterists' Bulletin. 27(1):33-37.

Gill, B. 1991. Dung beetle in tropical american forest. In Hanski, I. y Y. Camberfort (eds.). Dung beetle ecology. Princeton University Press. Princeton. Nueva Jersey. p 210-227.
Giraldo, C., F. Escobar. J. Chara y Z. Calle. 2011. The adoption of silvopastoral systems promotes the recovery of ecological processes regulated by dung beetles in the Colombian Andes. Insect Conservation and Diversity 4(2):115-122.

Gotelli, N. y R. Colwell. 2011. Estimating species richness. Biological diversity: frontiers in measurement and assessment. p 39-54.

Gotelli, N. y G. Entsminger. 2001. EcoSim: Null models software for ecology.

Halffter, G. 1997. Subsocial behavior in Scarabaeinae beetles. In Choe, J y B. Crespi (eds.) The evolution of social behavior in insects and arachnids. Cambridge University Press, Cambridge. p 142-157.

Halffter, G., M. Favila y V. Halffter. 1992. A comparative study of the structure of the scarab guild in Mexican tropicals rain forest and derived ecosystems. Folia Entomologica Mexicana 0(84):131-156.

Halffter, G. y E Matthews, E. 1966 The natural history of dung beetles of the subfamily Scarabaeinae Coleoptera, Scarabaeidae. Folia Entomologica Mexicana 12:1-312.

Halffter, G., C. Moreno y E. Pineda. 2001. Manual para evaluación de la biodiversidad en Reservas de la Biosfera. M\&T-Manuales y Tesis SEA, vol. 2. Zaragoza. $82 \mathrm{p}$.

Hammer, Ø., Harper, D. y Ryan, P. 2001. PAST: Paleontological Statistics Software Package for Education and Data Analysis. Palaeontología Electrónica 4(1): $1-9$.

Hanski, I y Y. Camberfort. 1991. Competition in dung beetles. In Hanski, I. y Y. Camberfort, (eds.). Dung beetle ecology. Princeton University Press, Princeton, Nueva Jersey. p 305-329.

Holland, S. 2008. Non-metric multidimensional scaling (mds). University of Georgia. Athens. file://C:/Users/Jose\%20Daniel/Documents/mdsTutorial. pdf. Recuperado el 18 de julio del 2014.

Horgan, F. 2001. Burial of bovine dung by coprophagous beetles (Coleoptera: Scarabaeidae) from horse and cow grazing sites in El Salvador. European Journal of Soil Biology 37(2):103-111.

Howden, F. y V. Nealis. 1975. Effects of clearing in a tropical rain forest on the composition of the coprophagous scarab beetle fauna (Coleoptera). Biotropica 7(2):77-83.

Hurlbert, S. 1971 The nonconcept of species diversity: a critique and alternate paradigms. Ecology 52(4): 577586. 
I.N.E. 2009. Uso del suelo en Honduras. INE, Honduras. http://www.ine.gob.hn/drupal/node/67. Recuperado el 12 de mayo del 2012.

Klein, B. 1989. Effects of forest fragmentation on dung and carrion beetle communities in central Amazonia. Ecology 70(6):1715-1725.

Kohlmann, B. 1996. The Costa Rican species of Ateuchus (Coleoptera: Scarabaeidae). Revista de Biología Tropical 44(3): 177-191.

Kohlmann, B. y A. Solís. 1997. El género Dichotomius (Coleoptera: Scarabaeidae) en Costa Rica. Giornale Italiano di Entomologia 8: 343-382.

Kohlmann, B. y A. Solís. 2001. El género Onthophagus (Coleoptera: Scarabaeidae) en Costa Rica. The genus Onthophagus (Coleoptera: Scarabaeidae) in Costa Rica. Giornale Italiano di Entomologia 9: 159-261.

Jessop, L. 1985. An identification guide to Eurysternine dung beetles (Coleoptera, Scarabaeidae). Journal of Natural History 19(6): 1087-1111.

Larsen, T. y A. Forsyth. 2005. Trap spacing and transect design for dung beetle biodiversity studies. Biotropica 37(2):322-325.

Legendre, P. y L. Legendre. 1998. Numerical ecology. Elsevier, Amsterdam, The Netherlands. 870 p.

Louzada, J., A. Lima, R. Matavelli, L. Zambaldi y J. Barlow. 2010. Community structure of dung beetles in Amazonian savannas: role of fire disturbance, vegetation and landscape structure. Landscape Ecology 25(4):631641.

Ludwig, J. y J. Reynolds. 1991. Statistical ecology: a primer on methods and computing, 1988. Willey, New York. $337 \mathrm{p}$.

Matthews, E. 1961. A revision of the genus Copris Müller of the western hemisphere (Coleoptera, Scarabaeidae). Entomologica Americana 41(1): 1-139.

Mejía, T. y P. House. 2002. Mapa de ecosistemas vegetales de Honduras. Manual de consulta. Proyecto P.A.A.R. Tegucigalpa. $60 \mathrm{p}$.

Navarrete, D. y G. Halffter. 2008. Dung beetle (Coleoptera: Scarabaeidae: Scarabaeinae) diversity in continuous forest, forest fragments and cattle pastures in a landscape of Chiapas, Mexico: the effects of anthropogenic changes. Biodiversity and Conservation 17(12):28692898.

Nichols, E., S. Spector, J. Louzada, T. Larsen, S. Amezquita y M. Favila. 2008. Ecological functions and ecosystem services provided by Scarabaeinae dung beetles. Biological Conservations 141(6):1461-1474.
Padilla, M., N. García y R. Hernández. 2009. Plan de desarrollo municipal con enfoque de ordenamiento territorial, Municipio de Yuscarán, El Paraíso. Forcuencas. Yuscarán. 50 p.

PAGS. 1998. Memoria taller técnico: "La contribución de los bosques latifoliados al desarrollo sostenible de Honduras". Proyecto de apoyo a la gestión sostenible de los recursos naturales de Honduras (PAGS). Tegucigalpa, M.D.C.

Quintero, I, y G. Halffter. 2009. Temporal changes in a community of dung beetles (Insecta: Coleoptera: Scarabaeinae) resulting from the modification and fragmentation of tropical rain forest. Acta Zoológica Mexicana 25(3):625-649.

R Core Team. 2013. R: a language and environment for statistical computing. $\mathrm{R}$ Foundation for Statistical Computing. Austria. Url: http://www.R-project.org.

Saleck, D. 2001. Caracterización del bosque seco de la comunidad de Oropolí, Honduras. Tesis Ing. Agr. Escuela Agrícola Panamericana, Zamorano, Honduras. $78 \mathrm{p}$.

Solís, A. y B. Kohlmann. 2002. El género Canthon (Coleoptera: Scarabaeidae) en Costa Rica. Giornale Italiano di Entomologia 10(50):1-68.

Spector, S., y A. Forsyth. 1998. Indicator taxa for biodiversity assessment in the vanishing tropics. In Conservation in a Changing World. Mace, M., A. Balmford y J. Ginsberg (eds.). Cambridge University Press, Cambridge, UK. p 181-209.

Vulinec, K. 2002. Dung beetle communities and seed dispersal in primary forestand disturbed land in Amazonia. Biotropica 34(2):297-309.

Yamada, D., O. Imura, K. Shi y T. Shibuya. 2007. Effect of tunneler dung beetles on cattle dung decomposition, soil nutrients and herbage growth. Grassland Science 53(2):121-129.

Verdú, J., L. Arellano y C. Numa. 2006. Thermoregulation in endothermic dung beetles (Coleoptera: Scarabaeidae): effect of body size and ecophysiological constraints in flight. Journal of Insect Physiology 52(8):854-860.

Vaz-de-Mello, F., W. Edmonds, F. Ocampo y P. Schoolmeesters. 2011. A multilingual key to the genera and subgenera of the subfamily Scarabaeinae of the new world (Coleoptera: Scarabaeidae). Zootaxa 2854: 1-73.

Recibido para publicación el 6 de junio de 2014.

Aceptado para publicación el 13 de septiembre de 2014. 